Article

\title{
Importance of Defluviitalea raffinosedens for Hydrolytic Biomass Degradation in Co-Culture with Hungateiclostridium thermocellum
}

\author{
Regina Rettenmaier ${ }^{1,+}$, Martina Schneider ${ }^{1,+}{ }^{\text {, Bernhard Munk }}{ }^{2} \mathbb{D}$, Michael Lebuhn ${ }^{2} \mathbb{D}$, \\ Sebastian Jünemann ${ }^{3,4}\left(\mathbb{D}\right.$, Alexander Sczyrba ${ }^{4}\left(\mathbb{D}\right.$, Irena Maus ${ }^{3, *(\mathbb{D}}$, Vladimir Zverlov ${ }^{1,5, *}$ \\ and Wolfgang Liebl ${ }^{1}$ (D) \\ 1 Chair of Microbiology, Technical University of Munich, Emil-Ramann-Str. 4, 85354 Freising, Germany; \\ regina.rettenmaier@tum.de (R.R.); martina.schneider@tum.de (M.S.); wliebl@wzw.tum.de (W.L.) \\ 2 Department for Quality Assurance and Analytics, Bavarian State Research Center for Agriculture, \\ Lange Point 6, 85354 Freising, Germany; bernhard.munk@lfl.bayern.de (B.M.); \\ michael.lebuhn@lfl.bayern.de (M.L.) \\ 3 Center for Biotechnology (CeBiTec), Universitätsstr. 27, 33615 Bielefeld, Germany; \\ jueneman@CeBiTec.Uni-Bielefeld.de \\ 4 Faculty of Technology, Bielefeld University, Universitätsstr. 25, 33615 Bielefeld, Germany; \\ asczyrba@techfak.uni-bielefeld.de \\ 5 Institute of Molecular Genetics, RAS, Kurchatov Sq. 2, 123182 Moscow, Russia \\ * Correspondence: irena.maus@cebitec.uni-bielefeld.de (I.M.); vladimir.zverlov@tum.de (V.Z.) \\ + These authors equally contributed to this work.
}

Received: 19 May 2020; Accepted: 11 June 2020; Published: 17 June 2020

\begin{abstract}
Bacterial hydrolysis of polysaccharides is an important step for the production of sustainable energy, for example during the conversion of plant biomass to methane-rich biogas. Previously, Hungateiclostridium thermocellum was identified as cellulolytic key player in thermophilic biogas microbiomes with a great frequency as an accompanying organism. The aim of this study was to physiologically characterize a recently isolated co-culture of $H$. thermocellum and the saccharolytic bacterium Defluviitalea raffinosedens from a laboratory-scale biogas fermenter. The characterization focused on cellulose breakdown by applying the measurement of cellulose hydrolysis, production of metabolites, and the activity of secreted enzymes. Substrate degradation and the production of volatile metabolites was considerably enhanced when both organisms acted synergistically. The metabolic properties of $H$. thermocellum have been studied well in the past. To predict the role of $D$. raffinosedens in this bacterial duet, the genome of $D$. raffinosedens was sequenced for the first time. Concomitantly, to deduce the prevalence of $D$. raffinosedens in anaerobic digestion, taxonomic composition and transcriptional activity of different biogas microbiomes were analyzed in detail. Defluviitalea was abundant and metabolically active in reactor operating at highly efficient process conditions, supporting the importance of this organism for the hydrolysis of the raw substrate.
\end{abstract}

Keywords: biogas; metagenomics; whole-genome sequencing; cellulose degradation; carbohydrate active enzymes; metabolic interaction

\section{Introduction}

The microbial conversion of lignocellulosic biomass to methane-rich biogas has become an important sustainable generating industry [1-3]. The process can be divided into several steps with different bacterial or archaeal organisms actively involved: (i) hydrolysis of the insoluble raw substrate by hydrolytic, saccharolytic, and peptolytic Bacteria; (ii) acidogenesis and acetogenesis by 
acidogenic and/or acetogenic Bacteria, including syntrophic oxidizers of acetate or other intermediates; and (iii) methanogenesis by methanogenic Archaea [4] with a diverse spectrum metabolic pathways for methanogenesis, mainly aceticlastic, hydrogenotrophic, or $\mathrm{H}_{2}$ and methylotrophic methanogenesis [5].

Integrated omics approaches describing biogas microbiology previously indicated the presence a huge fraction of unassignable sequences, suggesting that most of the microorganisms in biogas communities are still unknown [6-10]. This is due to insufficient availability of reference strains and their corresponding genome sequences in public databases. Addressing this issue, Maus et al. [11] isolated and genetically characterized novel cellulolytic, hydrolytic, and acidogenic/acetogenic Bacteria as well as methanogenic Archaea originating from different anaerobic digestion communities. This study and earlier work $[9,11]$ showed that (hemi)cellulolytic Bacteria mostly represented a minority within the entire biogas microbiome despite their unquestionably pivotal role in initial biomass breakdown, which can be crucial and rate-limiting for the entire biogas process $[4,12]$. Therefore, it is postulated that few true cellulolytic Bacteria cooperate intimately and synergistically along with saccharolytic and synthrophic Bacteria [13-15]. Such cross-feeding has already been reported, e.g., for cellulolytic Bacteria of the genus Hungateiclostridium [16], formerly known as Clostridium [17-19].

Previous analysis of biogas-producing microbial communities revealed, beside $H$. thermocellum as truly cellulolytic organism, many saccharolytic organisms such as Defluviitalea spp. accounting for $90 \%$ of the relative abundance in cellulolytic mixed isolates or up to $34 \%$ in cellulolytic enrichment cultures derived from thermophilic lab-scale biogas fermenters [20]. To unravel the effect of the $H$. thermocellum cellulolytic activity in co-culture with D. raffinosedens, the strain D. raffinosedens $249 \mathrm{c}-\mathrm{K} 6$ was isolated in pure culture and its genomic sequence was characterized in detail. This is the first genome of the species D. raffinosedens and the second genome of the genus Defluviitalea sequenced so far. Further, results of targeted metagenomic and metatranscriptomic sequence analysis are integrated to examine the role of $D$. raffinosedens in the anaerobic biomass decomposition process.

\section{Materials and Methods}

\subsection{Data Availability}

The genome assembly of $D$. raffinosedens $249 \mathrm{c}-\mathrm{K} 6$ is available at NCBI under Accession No. WSLF00000000 with the Bioproject Accession No. PRJNA591875. Additionally, the raw sequencing reads of the whole genome sequencing were deposited at NCBI with Accession No. SRR10673227. Further, the almost complete $16 \mathrm{~S}$ rRNA gene sequence of D. raffinosedens 249c-K6, as established via direct, gene-specific PCR and Sanger sequencing, is available with Accession No. MN744427. The full-length $16 \mathrm{~S}$ rRNA gene sequence of $D$. raffinosedens $249 \mathrm{c}-\mathrm{K} 6$, assembled from the genome sequence and the gene-specific PCR, is available at NCBI with Accession No. MT350287. The sequence datasets of the $16 \mathrm{~S}$ rRNA amplicon libraries are available in the ENA repository under the Bioproject ID PRJEB37872.

\subsection{Reactor Operation and Sampling}

In this study, two mesophilic $\left(38^{\circ} \mathrm{C}\right)$ and two thermophilic $\left(50^{\circ} \mathrm{C}\right)$ laboratory-scale biogas fermenters (44 L working volume) fed with maize silage only were investigated at different process conditions. Two reactors were operated in parallel for each temperature. All fermenters were started up with inocula from an industrial-scale biogas plant located in Eschelbach an der Ilm, Germany, digesting a feedstock of $72 \%$ maize silage, $21 \%$ rye whole-crop silage, $3 \%$ chicken manure, and small amounts of cow dung, corn cob mix, and grass silage. Inocula were taken from the secondary reactor (operated at $44^{\circ} \mathrm{C}$ ) or the main fermenter (operated at $46^{\circ} \mathrm{C}$ ) for mesophilic or thermophilic fermenter operation, respectively. Detailed analyses of inocula are summarized in Supplementary Table S1.

After stable anaerobic digestion and biogas production conditions were reached, the processes were transformed to an acidified (mesophilic) or highly efficient (thermophilic) status. Processes are defined stable if no accumulation of volatile fatty acids (VFAs) was observed, and if the methane yield 
laid in an expected range of 350-400 $\mathrm{LSTP}_{\mathrm{ST}} \mathrm{CH}_{4} \times \mathrm{kg}_{\mathrm{VS}}{ }^{-1}$ for the digestion of maize silage. The process was stable in the thermophilic reactors (t_st) at an OLR of $2.0 \mathrm{~kg}_{\mathrm{vs}} \times \mathrm{m}^{-3} \times \mathrm{day}^{-1}$. The OLR was increased, but VFAs did not accumulate, and the methane yield still ranged $350-400 \mathrm{~L}_{\mathrm{STP}} \mathrm{CH}_{4} \times \mathrm{kg}_{\mathrm{VS}}{ }^{-1}$ at $4.0 \mathrm{~kg}_{\mathrm{VS}} \times \mathrm{m}^{-3} \times \mathrm{day}^{-1}$. This status was defined as highly efficient ( $\left.\mathrm{t} \_\mathrm{ef}\right)$. In the mesophilic reactors, the process was stable and efficient (m_st) at an OLR of $2.5 \mathrm{~kg}_{\mathrm{Vs}} \times \mathrm{m}^{-3} \times \mathrm{day}^{-1}$. VFAs accumulated here in the following, and the methane yield dropped below $300 \mathrm{LSTP}_{\text {ST }} \mathrm{CH}_{4} \times \mathrm{kgVS}^{-1}$. This disturbed process was defined as acidified (m_ac). Samples were taken from $t \_s t, t \_e f, m \_s t$, and $m \_a c$ processes for conventional biochemical and microbial community analyses (see below). Fresh samples were processed without delay in the adjacent laboratory.

\subsection{Organisms}

The consortia GS2.5GR_T4 and GS2_O45 originate from a fermenter operation $\left(55^{\circ} \mathrm{C}\right)$ ahead of $t \_s t$. Sampling, enrichment, and preliminary identification of these consortia were described by Rettenmaier et al. [20]. Separation of clonal pure isolates from these cultures was performed as described previously [20] or by standard streaking techniques on agar plates and re-inoculation of single colonies in liquid media. Isolate 250c-K4 (H. thermocellum) originates from GS2_O45, while the co-culture 253-K6 (H. thermocellum and D. raffinosedens) and isolate 249c-K6 (D. raffinosedens) originate from GS2.5GR_T4. H. thermocellum DSM $1237^{\mathrm{T}}=$ ATCC $27405^{\mathrm{T}}$ was purchased from the German Collection of Microorganisms and Cell Cultures (DSMZ, Braunschweig, Germany).

\subsection{Growth Conditions Used for D. raffinosedens $249 c-K 6$ and H. thermocellum DSM $1237^{T}$}

During this study, the three different cultivation media used for this study were: (i) tap water with 20\% (v/v) sterilized biogas plant digestate, designated GR20; (ii) GS2 medium [21,22]; and (iii) GS2 supplemented with 2.5\% (v/v) digestate, designated GS2.5GR, as also described previously [20]. Filter paper (FP) (Whatmann No.1), crystalline cellulose powder (MN301; Machery Nagel; Düren, Germany), glucose, or xylose was added at $0.2-0.5 \%(w / v)$. All experimental steps were performed under anaerobic atmosphere $\left(98 \% \mathrm{~N}_{2}, 2 \% \mathrm{H}_{2}\right)$ in an anaerobic chamber (Coy Laboratory Products, Grass Lake, MI, USA). Liquid medium was prepared in anaerobic flasks closed with a butyl-rubber stopper. For solid medium, 1.8\% (w/v) agar was added before autoclaving. Agar plates were incubated anaerobically in AnaeroJars (Thermo Fisher Scientific, Waltham, MA, USA). Ten milliliters of liquid medium were inoculated 1:100 and $50 \mathrm{~mL}$ medium for Anthrone assay, gas chromatography (GC), and the determination of enzymatic activities were inoculated 1:50. All cultures were incubated at $55^{\circ} \mathrm{C}$.

\subsection{Determination of Cellulose Hydrolyzing Activities by the Anthrone Assay of H. thermocellum Alone or in Co-Culture with D. raffinosedens}

Cellulolytic cultures consisting of $H$. thermocellum alone or in co-culture with $D$. raffinosedens were pre-cultivated in $10 \mathrm{~mL}$ liquid medium (GS2, GS2.5GR, or GR20) with FP at $55^{\circ} \mathrm{C}$. The incubation time varied between two and eight days depending on the cellulolytic property of the culture. One milliliter of the pre-culture was inoculated in $50 \mathrm{~mL}$ liquid medium with $0.2 \%(w / v) \mathrm{MN} 301$ as biological duplicates and incubated at $55{ }^{\circ} \mathrm{C}$. Two to eight days after inoculation, aliquots of $2-4 \mathrm{~mL}$ were centrifuged (13,000 rpm, $5 \mathrm{~min})$. Pellets were washed twice in $1 \mathrm{~mL}$ deionized water $\left(\mathrm{MQ} \mathrm{H}_{2} \mathrm{O}\right)$, once in $1 \mathrm{~mL} \mathrm{100 \%}$ ethanol, and dried. For swelling of cellulose, $175 \mu \mathrm{L} 72 \%(v / v) \mathrm{H}_{2} \mathrm{SO}_{4}$ was added and incubated for $1 \mathrm{~h}$ at room temperature (RT). Samples were filled up to $1 \mathrm{~mL}$ with $\mathrm{MQ} \mathrm{H}_{2} \mathrm{O}$ and hydrolyzed for $30 \mathrm{~min}$ at $100{ }^{\circ} \mathrm{C}$. For glucose assays, samples representing the glucose standard curve $\left(0.02-0.2 \mathrm{mg} \cdot \mathrm{mL}^{-1}\right)$ as well as dilutions of the hydrolyzed cellulose samples (1:4/1:10/1:20/1:40) were prepared with $\mathrm{MQ} \mathrm{H}_{2} \mathrm{O}$ in a flat-bottom 96-well plate on ice. Two hundred milliliters of ice-cooled Anthrone $0.2 \%(w / v)$ in $96 \%(v / v) \mathrm{H}_{2} \mathrm{SO}_{4}$ (both Carl Roth, Karlsruhe, Germany) were added to each well, and, subsequently, the plate was incubated in a water bath at $80^{\circ} \mathrm{C}$ for $30 \mathrm{~min}$. Afterwards, 
the absorption was measured at $620 \mathrm{~nm}$ with a SPECTROstarnano (BMG LABTECH, Ortenberg, Germany) or Tecan Infinite M200 PRO (Tecan Group Ltd., Männedorf, Switzerland) photometer.

\subsection{Determination of Volatile Acids and Alcohols with Gas Chromatography}

To detect the amount of volatile acids and alcohols in the sample, GC analysis was performed using the GC-2010 system (Shimadzu, Kyoto, Japan) with a flame ionization detector as described previously [22]. In brief, samples were separated with a Stabilwax-DA capillary column (RESTECK, Bad Homburg, Germany) and nitrogen as gas carrier $\left(50 \mathrm{~cm} \times \mathrm{s}^{-1}\right)$. A temperature gradient $\left(12 \mathrm{~min}\right.$ from $70{ }^{\circ} \mathrm{C}$ to $260{ }^{\circ} \mathrm{C}$ followed by $2 \mathrm{~min}$ at $260{ }^{\circ} \mathrm{C}$ ) was used for more effective separation. Two milliliters of fresh liquid culture was centrifuged ( $3 \mathrm{~min}, 13,000 \mathrm{rpm}$ ), and the supernatant was stored at $-20{ }^{\circ} \mathrm{C}$ until measurement. Thawed samples were centrifuged again (20 min, 13,000 rpm) and $100 \mu \mathrm{L}$ of the supernatant was mixed with $350 \mu \mathrm{L} \mathrm{H}_{2} \mathrm{O}(\mathrm{pH} 2.0$ with $\mathrm{HCl}$ ) and $50 \mu \mathrm{L} 0.5 \%(v / v)$ 1-propanol as internal standard (final concentration $0.05 \%(v / v)$ ). For identification of peaks and quantification of products, an external standard with methanol, ethanol, isopropyl, butanyl, isobutanyl, butyric acid, acetic acid, and propionic acid ( $0.5 \%(v / v)$ each) was used.

\subsection{Enzymatic Digestion of Cellulose Biomass and Determination of Enzymatic Activity}

Bacterial cells were removed from fresh liquid cultures via centrifugation (4500 rpm, $30 \mathrm{~min}$, $4{ }^{\circ} \mathrm{C}$ ). Under continuous stirring, saturated ammonium sulfate solution was added in little droplets to the culture supernatant at $4{ }^{\circ} \mathrm{C}$ to a final concentration of $60 \%(v / v)$ ammonium sulfate. The solution was stirred over night at $4{ }^{\circ} \mathrm{C}$. Precipitated proteins were centrifuged $\left(4500 \mathrm{rpm}, 30 \mathrm{~min}, 4{ }^{\circ} \mathrm{C}\right)$, washed once in $20 \mathrm{~mL} 0.1 \mathrm{M}$ MOPS buffer (0.1 M MOPS, $0.05 \mathrm{M} \mathrm{NaCl}, 0.01 \mathrm{M} \mathrm{CaCl}_{2}, \mathrm{pH} 7.0$ ) with $60 \%(v / v)$ ammonium sulfate, and centrifuged again $\left(4500 \mathrm{rpm}, 20 \mathrm{~min}, 4{ }^{\circ} \mathrm{C}\right)$. All proteins were stored in a total volume of $5 \mathrm{~mL} 0.1 \mathrm{M}$ MOPS buffer with $60 \%(v / v)$ ammonium sulfate at $4{ }^{\circ} \mathrm{C}$. For renaturation, $1 \mathrm{~mL}$ of the solution with the precipitated proteins was centrifuged ( $3 \mathrm{~min}, 13,000 \mathrm{rpm}$ ), and the pellet was dissolved in $500 \mu \mathrm{L}$ buffer (1× MOPS, $\mathrm{pH}$ 7.0) over night on ice. The protein concentration was determined by $\mathrm{A}_{280}$ measurement by a spectrophotometer (Eppendorf, Hamburg, Germany). Enzymatic activities on barley- $\beta$-glucan (BBG), phosphoric acid swollen cellulose (PASC), and cellulose powder MN301 were measured by liberation of reducing sugars [23], as described previously [22]. In brief, all protein-substrate combinations were incubated as biological duplicates in reactions containing $100 \mu \mathrm{L} 1 \%(w / v)$ substrate (BBG, MN301, PASC), $15 \mu \mathrm{L}$ buffer (1× MOPS, $\mathrm{pH} 7.0), 0.1 \mu \mathrm{g} \times \mu \mathrm{L}^{-1}$ proteins, and filled up to a final reaction volume of $200 \mu \mathrm{L}$ with $\mathrm{MQ} \mathrm{H}_{2} \mathrm{O}$. The reaction tubes were incubated in a water bath at $55^{\circ} \mathrm{C}$ for $30 \mathrm{~h}$. The enzymatic digestion was stopped on ice and remaining substrate was removed by centrifugation. Fifty microliters digest samples or glucose standard samples $\left(0.1-2.0 \mathrm{mg} \cdot \mathrm{mL}^{-1}\right)$ were mixed as technical triplicates with $75 \mu \mathrm{L}$ DNSA solution (10 g. $\mathrm{L}^{-1}(w / v)$ 3,5-Dinitrosalicylic acid (DNSA), $10 \mathrm{~g} \cdot \mathrm{L}^{-1}(w / v) \mathrm{NaOH}, 200 \mathrm{~g} \cdot \mathrm{L}^{-1}(w / v)$ sodium-potassium tartrate, $0.5 \mathrm{~g} \cdot \mathrm{L}^{-1}(w / v)$ sodium sulfate, and $2.0 \mathrm{~g} \cdot \mathrm{L}^{-1}(w / v)$ phenol) in a deep 96-well PCR plate. Plates were incubated at $95^{\circ} \mathrm{C}$ in a thermoblock for $5 \mathrm{~min}$ and cooled on ice. The absorption of $100 \mu \mathrm{L}$ solution was measured in a flat-bottom 96 -well plate at $540 \mathrm{~nm}$ with a Sunrise ${ }^{\mathrm{TM}}$ photometer (Tecan Group Ltd., Männedorf, Switzerland).

\subsection{Species Identification, Sequencing, and Annotation of D. raffinosedens, Isolate $249 c-K 6$}

For species identification of the isolates obtained after cultivation, genomic DNA was extracted from $1 \mathrm{~mL}$ corresponding bacterial culture using the Soil DNA Extraction Kit (Roboklon, Berlin, Germany, manufacturer's instructions). The 16S rRNA gene was amplified and analyzed as described previously [20]. Presence of $H$. thermocellum was verified via species-specific primers (CthF: TTTACCGGAAGTATATCCTAG and CthR: AATCATCTGCCCCACCTTC), as described previously [24].

For extraction of D. raffinosedens 249c-K6 high molecular weight genomic DNA, a fresh overnight culture, grown in GS2 medium at $55^{\circ} \mathrm{C}$, was pelleted $\left(10 \mathrm{~min}, 5000 \mathrm{rpm}, 8^{\circ} \mathrm{C}\right)$ and washed twice in 
$5 \mathrm{~mL} 0.9 \%(w / v) \mathrm{NaCl}$ solution. The pellet was resuspended in $5 \mathrm{~mL}$ lysis buffer $(20 \mathrm{mM}$ Tris/HCl pH 7.5; 25 mM EDTA; 75 mM NaCl, 1 mg $\times \mathrm{mL}^{-1}$ lysozyme (AppliChem, Darmstadt, Germany)) and incubated for $1 \mathrm{~h}$ in a water bath at $37^{\circ} \mathrm{C}$. After adding $500 \mu \mathrm{L} 10 \%(w / v)$ SDS, $500 \mu \mathrm{L}$ proteinase $\mathrm{K}\left(10 \mathrm{mg} \times \mathrm{mL}^{-1}\right.$; AppliChem, Darmstadt, Germany) and $50 \mu \mathrm{L}$ RNase A (AppliChem, Darmstadt, Germany; DNase free), the solution was incubated at slow agitation for $2 \mathrm{~h}$ at RT. Then, $2 \mathrm{~mL} \mathrm{NaCl}$ $(5 \mathrm{M})$ and $3 \mathrm{~mL}$ chloroform/isoamyl alcohol (Roth) were added followed by incubation with slow agitation at RT for $30 \mathrm{~min}$. After centrifugation $\left(10 \mathrm{~min}, 5000 \mathrm{rpm}, 20^{\circ} \mathrm{C}\right)$, the upper liquid phase was transferred into a fresh falcon tube. After addition of $3 \mathrm{~mL}$ chloroform/isoamyl alcohol (Carl Roth, Karlsruhe, Germany), the solution was incubated for another 30 min with slow agitation at RT and centrifuged $\left(10 \mathrm{~min}, 5000 \mathrm{rpm}, 20^{\circ} \mathrm{C}\right)$. The upper liquid phase was transferred into a fresh falcon tube. These three steps were repeated until a clear phase separation was visible. The upper liquid phase was transferred again into a fresh falcon tube. Subsequently, DNA was precipitated by adding one volume of $100 \%(v / v)$ isopropanol (RT) and gentle agitation. Under a PCR hood, the high molecular weight DNA was grasped with a pipette tip and immersed two times in $500 \mu \mathrm{L}$ cold $\left(-20{ }^{\circ} \mathrm{C}\right) 70 \%(v / v)$ ethanol. The dried DNA was dissolved in $100 \mu \mathrm{L}$ MQ $\mathrm{H}_{2} \mathrm{O}$.

For genome sequencing of D. raffinosedens isolate $249 \mathrm{c}-\mathrm{K} 6$ at the ZIEL-Institute for Food \& Health, Core Facility Microbiome/NGS, Technical University of Munich, Freising, Germany, $1 \mu \mathrm{g}$ chromosomal DNA of a clonal pure culture was used to prepare a DNA library using the TruSeq DNA PCR-free sample preparation kit (Illumina, Inc., San Diego, CA, USA). A protocol optimized for DNA shearing and fragment size selection was applied [25]. The library was sequenced using the Illumina HiSeq system with about 3.2 million $2 \times 150$ base pairs (bp) reads in paired-end (PE) mode, according to the manufacturer's instructions. The raw sequencing data were assembled using SPAdes v. 3.11.1 [26]. Default parameters were used for all software unless specified otherwise.

The NCBI Prokaryotic Genome Annotation Pipeline [27] was used for prediction and annotation of open reading frames (ORFs) in the D. raffinosedens $249 \mathrm{c}-\mathrm{K} 6$ genome. Subsequently, all automatically annotated protein sequences of the whole genome sequence were annotated to $\mathrm{K}$ numbers with the BlastKOALA tool [28] specifying family or genus of nearest related organism (taxid: 1185408). Metabolic pathways were reconstructed and analyzed via the Kyoto Encyclopedia of Genes and Genomes (KEGG) database webpage (https://www.genome.jp/kegg/) using the KEGG mapper tool [29]. Additionally, carbohydrate-active enzymes (CAZymes) were annotated with the dbCAN2 web server (National Science Foundation; http://bcb.unl.edu/dbCAN2/blast.php) [30] utilizing HMMER [31] and DIAMOND [32], including the prediction of signal peptides via SignalP 4.0 [33]. Enzymatic function of automatically predicted CAZymes of special interest, here glycoside hydrolases with signal peptide, were checked manually with the Carbohydrate Active Enzymes (CAZy) database (http://www.cazy.org/) [34].

For phylogenetic analysis on the whole-genome level, the draft genome was compared with a multiple alignment of 92 up-to-date bacterial core genes (UBCG) derived from 1492 species covering 28 phyla using the UBCG pipeline version 3 on the EZBioCloud webpage (https://www.ezbiocloud.net/tools/ubcg) [35]. Our application of UBCG, in essence, first extracts core genes from the genomes using Prodigal 2.6.3 and hmmsearch 3.1b2, aligns them using Mafft v. 7.310, and finally infers phylogenetic trees for each gene and the concatenated alignment of all genes using Fasttree v. 2.1.10. Reference genomes for phylogenetic analysis were chosen by performing a comparison of the $16 \mathrm{~S}$ rRNA gene sequence from $D$. raffinosedens $249 \mathrm{c}-\mathrm{K} 6$ with the nucleotide BLAST tool (BLASTn) against the Reference RNA sequences (refseq_rna) database of the NCBI (National Center for Biotechnology Information, http://www.ncbi.nlm.nih.gov) using the Megablast algorithm. From the top 20 hits, the whole genome sequences, if available at the NCBI, were downloaded and used for comparison. Further, the average nucleotide identity (ANI) was calculated via jspecies.org and the ANIb algorithm [36]. 
2.9. Prevalence and Activity of D. raffinosedens in Mesophilic and Thermophilic Biogas Fermenters Applying $16 S$ rRNA Gene Amplicon Sequencing

To investigate the prevalence and activity of $D$. raffinosedens $249 \mathrm{c}-\mathrm{K} 6$ in the four different reactor samples (m_st, $m \_a c, t \_s t$, and $\left.t \_e f\right)$, nucleic acids were extracted in parallel (two replicates, numbers 1 and 2, for each reactor type) using the GeneMATRIX Universal DNA/RNA/Protein Purification Kit (EurX, Gdansk, Poland). The sample was washed twice with 0.85\% $\mathrm{KCl}$ [37] and $200 \mu \mathrm{L}$ of each of the washed sample were mixed with $300 \mu \mathrm{L}$ phenol and bead-beating in a Lysing Matrix E tube (MP Biomedicals, Eschewege Germany) for $2 \times 20 \mathrm{~s}$ at level 5.0 in the FastPrep-24 Instrument (MP Biomedicals, Eschewege Germany). Two hundred microliters of Lyse All buffer (and $10 \mu \mathrm{L}$ mercaptoethanol per mL buffer) and $300 \mu \mathrm{L}$ of DRP buffer (and $10 \mu \mathrm{L}$ mercaptoethanol per mL buffer) were added to $100 \mu \mathrm{L}$ of the upper aqueous supernatant and transferred to a DNA binding spin-column and centrifuged. The flow-through was mixed with $300 \mu \mathrm{L}$ ethanol $(100 \%(v / v))$ and applied to an RNA binding spin-column. Then, the extraction of RNA and DNA was performed according to the manufacturer's protocol. The RNA was finally eluted in $60 \mu \mathrm{L}$ RNase-free water and the DNA in $100 \mu \mathrm{L}$ Elution Buffer. From $30 \mu \mathrm{L}$ of the extracted RNA, possible DNA contamination was removed using the Turbo DNA-free Kit (Thermo Fisher Scientific, Waltham, MA, USA). The DNA-free RNA ( $5 \mu \mathrm{L})$ was reverse transcribed into cDNA using Multi-Temp Affinity Script Reverse Transcriptase (Agilent) and the 16S rRNA gene-specific reverse primer R1378 (5'-3' reverse primer CGGTGTGTACAAGGCCCGGGAACG). Adapter sequences, TCGTCGGCAGCGTCAGATGTGTATAAGAGACAG (forward) and GTCTCGTGGGCTCGGAGATGTGTATAAGAGACAG (reverse), were attached to the gene specific primers F939 (5'-3' forward primer GAATTGACGGGGGCCCGCACAAG) and R1378 targeting the 16S rRNA gene region V6-V8 of Bacteria [38]. With these modified primers, PCR was performed using Platinum Taq Polymerase (Thermo Fisher Scientific, Waltham, MA, USA) and running 25 cycles for amplification. After checking the amplicons on an agarose gel for the correct length they were sent to the ZIEL (Institute for Food \& Health, Core Facility Microbiome at the Technical University of Munich) for further processing and sequencing via "paired-end sequencing by synthesis" on an Illumina MiSeq system with a sequencing length of $2 \times 300$ bp PE, following the manufacturer's instructions.

Processing of raw MiSeq forward and reverse PE reads was done as described by Engel et al. [39] with the following minor adjustments to individual bioinformatic steps. To achieve a higher assembly rate, we assembled Miseq PE reads in an iterative manner using Flash v. 1.2.11 [40]. All reads failing the first round of read assembly were clipped to a q20 average quality threshold using sickle v. 1.33 [41] and re-submitted to flash. This process was repeated consecutively while increasing the quality clipping threshold by 3 up to the point where either all reads could be assembled or a maximum quality clipping threshold of q35 was reached. All other steps, i.e. adapter clipping with cutadapt v1.18 [42]; de-replication, alignment, filtering, and de-noising with mothur v. 1.41 .3 [43]; chimera checking and OTU clustering with USEARCH v. 8.0.1477 [44]; and taxonomic classification on genus level based on a naïve Bayesian classifier with a $80 \%$ confidence cutoff and the full SILVA database v. 132 [45], were carried out as described in detail previously [39] but without applying a length filtering step after primer clipping. Afterwards, consensus sequences of resulting OTUs were aligned with the structural rRNA aligner SSU-ALIGN v. 0.1.1 [46] and the alignment was used for reconstructing the best maximum-likelihood phylogeny from 100 rapid bootstrap replicates using the RAxML v. 8.0 [47] with the GTRCAT model. Subsequently, statistical analysis of the $16 \mathrm{~S}$ rDNA and reverse transcribed 16S rRNA amplicon sequencing was performed via RHEA [48] in R. 
2.10. Occurrence of D. raffinosedens Sequences in Biogas-Producing Microbial Communities Deduced from $16 S$ rDNA and Reverse Transcribed $16 S$ rRNA Amplicons of this Study or from Publicly Available Metagenome Data

For a targeted identification of the organisms of interest, namely D. raffinosedens 249c-K6, in the DNA and cDNA amplicon reads, PE assembled and primer clipped reads were mapped with the aln and samse module of the BWA aligner v. 0.7.13 [49] against the EzBioCloud database v. 2018.05 [50]. Mappings were filtered to retain mapped reads with a minimum mapping length of 300 bases and a minimum percent identity of $99 \%$ to the reference (full length $16 \mathrm{~S}$ rRNA gene sequence of D. raffinosedens 249c-K6 accession no MT350287) based on the CIGAR, NM, and XM tag of the resulting SAM file using SAMtools v. 1.8 [51] and custom scripts.

Additionally, publicly available metagenomic and metatranscriptomic datasets were analyzed for sequence identity to evaluate the occurrence of the D. raffinosedens 249c-K6 in biogas plants in general. The assembled genome of D. raffinosedens 249c-K6 was uploaded to the Microbial Genomes Atlas (http://microbial-genomes.org) [52] and compared to the project "biogas microbiome" described by Campanaro et al. [53] with default settings.

\section{Results and Discussion}

\subsection{Isolation of D. raffinosedens from Cellulolytic Mixed Cultures Isolated from Biogas Fermenters}

The overall aim of this study was to study and characterize the synergism of a hydrolytic/cellulolytic bacterial consortium isolated from biogas fermenters. Therefore, cellulolytic mixed cultures from a previous study [20] were examined in more detail. In this study, H. thermocellum was isolated repeatedly from these cultures (data not shown). One representative isolate, $H$. thermocellum strain 250c-K4, was further analyzed. Interestingly, from mixed cultures dominated by Defluviitalea and Hungateiclostridium spp. [20], the separation of Defluviitalea spp. in pure, cellulolytic cultures was not possible by standard methods. These attempts resulted again in co-cultures dominated by $D$. raffinosedens and, in minor amounts, $H$. thermocellum, as verified by $16 \mathrm{~S}$ rRNA gene sequencing and species-specific PCR, respectively (data not shown). The frequency of isolating $D$. raffinosedens growing in co-culture with $H$. thermocellum on crystalline cellulose also pointed to a putative synergism of these two organisms. As a representative for a co-culture of D. raffinosedens and H. thermocellum, the consortium 253c-K6 was further analyzed.

Pure cultures of Defluviitalea spp. were obtained by changing the carbon source from crystalline cellulose to xylose and carrying out several transfers and cultivation in liquid medium before isolating single colonies from streak plates and re-inoculation of single colonies in liquid media. This approach was successful apparently because the saccharolytic species $D$. raffinosedens is able to use different monoand disaccharides including pentoses such as xylose as carbon source [54], whereas H. thermocellum is not able to use pentoses for growth [55]. Subsequently, the clonal pure cultures were identified as belonging to $D$. raffinosedens showing $99.71 \% 16 \mathrm{~S}$ rRNA gene sequence identity to $D$. raffinosedens, $\mathrm{A}^{\mathrm{T}}{ }^{\text {[54] }}$ and 96.29\% identity to D. saccharophila, LIND6LT2 ${ }^{\mathrm{T}}$ [56]. The top 20 list showing the comparison of the 16S rRNA gene sequence of the isolate $D$. raffinosedens against the NCBI database are summarized in Supplementary Table S2. As a representative isolate, strain 249c-K6 was further analyzed and its $16 \mathrm{~S}$ rRNA gene sequence was deposited at NCBI database under the accession number MN744427.

\subsection{Genome Sequence Analysis of the Strain D. raffinosedens $249 c-K 6$}

To investigate the effect of the $H$. thermocellum cellulolytic activity in co-culture with $D$. raffinosedens, the genome of the strain D. raffinosedens 249c-K6 was sequenced and analyzed in detail. This is the first genome of the species $D$. raffinosedens sequenced thus far. The assembly of the whole genome sequencing resulted in 31 contigs ( $\geq 1000 \mathrm{bp}$ ) with a total length of 3,092,142 bp and an average 70-fold sequencing coverage. The average G + C content was $35.67 \mathrm{~mol} \%$. Gene prediction and manual annotation of the D. raffinosedens $249 \mathrm{c}-\mathrm{K} 6$ genome sequence were performed applying the 
NCBI Prokaryotic Genome Annotation Pipeline [27] and resulted in the identification of 2,950 genes in total, thereof 2806 protein coding and 58 pseudo genes. Further, 86 RNA genes encoding 3 5S rRNAs, $716 \mathrm{~S}$ rRNAs, $523 \mathrm{~S}$ rRNAs, 67 tRNAs, and 44 non-coding RNAs were predicted. The genome sequence of D. raffinosedens 249c-K6 was deposited at NCBI database under the accession number PRJNA591875.

\subsubsection{Phylogenetic Classification of D. raffinosedens 249c-K6 in Relation to Members of the} Genus Defluviitalea

Among the Defluviitalea members previously described in the literature [54,56,57], only for the relative strain D. phaphyphila Alg1 [57] complete genome sequence information is publicly available. Strains D. raffinosedens 249c-K6 and D. phaphyphila Alg1 showed an ANI value of 75.61\% with 40.84\% of the $249 \mathrm{c}-\mathrm{K} 6$ genome aligned to the genome of D. phaphyphila, indicating that strain $249 \mathrm{c}-\mathrm{K} 6$ belongs to another species distinct of D. phaphyphila [58,59]. Thus, the genome sequence of strain 249c-K6 provided here helps to elucidate the genome diversity within this genus. To determine the phylogeny of $D$. raffinosedens $249 \mathrm{c}-\mathrm{K} 6$ in relation to other completely sequenced closely related members, as determined by comparison of the 249c-K6 16S rRNA gene sequence (Accession No. MN744427) analysis against the NCBI database (refsequ_rna), 14 reference strains including D. phaphyphila Alg1 were used to build the phylogenetic tree. The reference strains studied, except D. phaphyphila Alg1, shared only 88-89\% 16S rRNA gene sequence identity to strain 249c-K6 (Supplementary Table S3). The phylogenetic tree demonstrated close relatedness of D. raffinosedens 249c-K6 to D. phaphyphila Alg1 supporting affiliation of the strain 249c-K6 to the genus Defluviitalea (Figure 1). Further, the UBCG tree sustains the phylogenetic distance to the reference strains already observed at 16S rRNA gene sequence level.

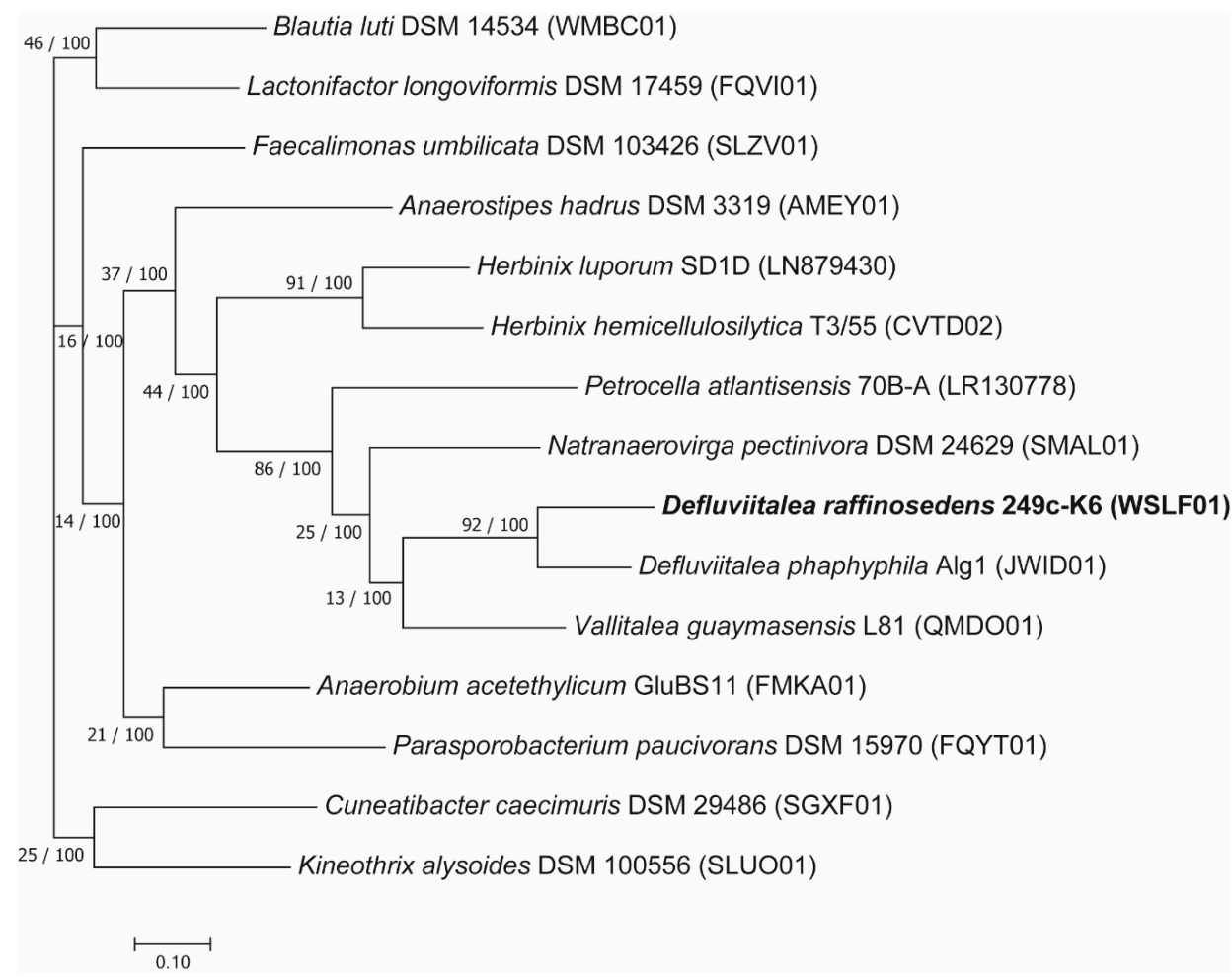

Figure 1. Phylogenetic position of D. raffinosedens isolate $249 \mathrm{c}-\mathrm{K} 6$ in relation to the most closely related members of the class Clostridia. The tree was constructed applying the UBCG pipeline v. 3 [35] using standard settings based on 92 up-to-date bacterial core genes. In total, 86,940 nucleotide positions were used to build the concatenated alignment. For all branches, the number of examined genes (GSIs, gene support indices) supporting the given topology and bootstrap values are shown as follows: GSI/Bootstrap value. Bar, 0.01 substitutions per position. 


\subsubsection{D. raffinosedens 249c-K6 Metabolic Pathways Predicted by Means of KEGG}

Reconstruction of metabolic pathways utilizing the BlastKOALA [28] and KEGG mapper tool [29] revealed a total of 37 complete pathway modules annotated in the draft genome of D. raffinosedens 249c-K6 (Supplementary Table S4). These included seven modules from the central carbohydrate metabolism: Glycolysis (Embden-Meyerhof pathway) (M00001), Glycolysis (M00002), Gluconeogenesis (M00003), Pyruvate oxidation (M00307), Citrate cycle (first carbon oxidation) (M00010), Pentose phosphate pathway, non-oxidative (M00007), and PRPP biosynthesis (M00005). Moreover, experimental physiological characterization revealed growth of 249c-K6 on xylose as carbon source (data not shown), which is consistent with the strain description of $D$. raffinosedens [54]. The pentose phosphate pathway is important for xylose utilization, as recently reported for Pseudoclostridium thermosuccinogenes [60]. Therefore, the presence of genes for the complete non-oxidative pentose phosphate pathway in the draft genome supports the experimentally observed xylose utilizing property of D. raffinosedens $249 \mathrm{c}-\mathrm{K} 6$.

\subsubsection{D. raffinosedens $249 \mathrm{c}-\mathrm{K} 6$ Genes Predicted to be Involved in Carbohydrate Utilization}

To gain a better insight into the saccharolytic potential of $D$. raffinosedens, the draft genome was analyzed for CAZyme-encoding genes with the dbCAN2 meta server [30] and revealed a total of 53 CAZymes including 32 glycoside hydrolases (GH), 12 glycosyl transferases (GT), 2 carbohydrate esterases (CE), and 7 multi-domain enzymes. Of all GHs, only for six a signal peptide (SP) was predicted. As GHs with SPs are of particular relevance for the extracellular degradation of complex plant biomass, they were examined manually for their enzymatic function in the CAZy database [34]. This revealed the presence of GH13 (subfamily 36), GH32 and GH43 (subfamilies 4 and 16), as well as carbohydrate-binding modules (CBM) 6 and 38. For one multi-domain protein [CBM38(44-189) + CBM38(221-367) + CBM38(409-527) + GH32(808-1121) + CBM38(1313-1448)], enzymatic activity on the fructose polysaccharide inulin might be possible. Enzymatic activities within the identified (sub-)families are summarized in Supplementary Table S5.

\subsection{Physiological Comparison of H. thermocellum as Pure Culture and in Co-Culture with D. raffinosedens}

To determine cellulose hydrolysis properties of $H$. thermocellum as pure culture in comparison to the co-culture with $D$. raffinosedens, strains were analyzed in three different media supplemented with cellulose powder MN301 as sole carbon source. The determination of cellulose-hydrolyzing activities by the Anthrone assay revealed higher activities, manifested as a larger extent of cellulose degradation, for 253c-K6, a co-culture of $H$. thermocellum and D. raffinosedens, compared to two pure cultures of H. thermocellum (ATCC27405 ${ }^{\mathrm{T}}$ and 250c-K4), independent of the medium used for cultivation (Figure 2A). Especially in GS2 and GS2.5GR medium, 99\% of MN301 was hydrolyzed by the co-culture within two days of cultivation at $55^{\circ} \mathrm{C}$. H. thermocellum 250c-K4 alone hydrolyzed only $84 \%$ MN301 in GS2 and 93\% in GS2.5GR within the same incubation period. As expected, D. raffinosedens 249c-K6 alone did not grow on cellulose as the sole carbon source. Similarly, higher amounts of total volatile metabolites and higher concentrations of acetic acid were detected by GC analysis for the co-culture 253c-K6, as compared to H. thermocellum, strains ATCC $27405^{\mathrm{T}}$ or $250 \mathrm{c}-\mathrm{K} 5$, alone (Figure $2 \mathrm{~B}$ ). In contrast, the specific activity of culture supernatant proteins on different $\beta$-glucans (soluble (BBG), crystalline (MN301), and amorphous (PASC)) did not differ much when $H$. thermocellum was in co-culture with D. raffinosedens (253c-K6) compared to $H$. thermocellum alone (strains ATCC $27405^{\mathrm{T}}$ or $250 \mathrm{c}-\mathrm{K} 5$ ) (Figure $2 \mathrm{C}$ ). All three cultures showed highest enzymatic activity on BBG $\left(1.8 \mathrm{mU} \times \mathrm{mL}^{-1}\right)$ and lowest on MN301 $\left(0.3-0.6 \mathrm{mU} \times \mathrm{mL}^{-1}\right)$. Thus, the co-culture of $D$. raffinosedens with $H$. thermocellum showed enhanced cellulose hydrolysis as well as metabolic activity. However, cellulolytic enzyme activities of culture supernatant proteins were not increased when $H$. thermocellum was co-cultivated with $D$. raffinosedens. This indicates that the observed enhanced cellulose hydrolysis of the co-culture is rather caused by an (metabolic) interaction of both organisms and not by extracellular enzymes of $D$. raffinosedens that might have cleaved crystalline 
cellulose. Besides, D. raffinosedens is described as a non-cellulolytic, but saccharolytic organism [54]. Furthermore, co-cultures of $H$. thermocellum with other, non-cellulolytic organisms have previously been found in the context of lignocellulose fermentation to ethanol [18]. For example, a co-culture of H. hermocellum and Thermoanaerobacter thermohydrosulfuricus was reported to ferment hemicellulose from Solka Floc, in contrast to both organisms as pure cultures [61]. The authors proposed that by a metabolic interaction of both organisms, xylobiose and xylose released by (hemi-)cellulase activity of $H$. thermocellum were fermented by $T$. thermohydrosulfuricus to ethanol. H. thermocellum breaks down cellobiose and cellodextrins intracellularly by phosphorolytic cleavage and utilizes only the formed glucose-1-phosphate, whereas glucose is liberated into the medium [62]. Thus, by growth on hemicellulose, pentose sugars liberated by $H$. thermocellum can be utilized via cross-feeding by other organisms. In the case of $H$. thermocellum in co-culture with $D$. raffinosedens, we suppose that the saccharolytic organism $D$. raffinosedens uses the liberated soluble sugars for its growth. These soluble sugars thereby are removed and cannot act as end-product inhibitors of $H$. thermocellum cellulases anymore, which plausibly explains the enhanced cellulose hydrolysis.

A

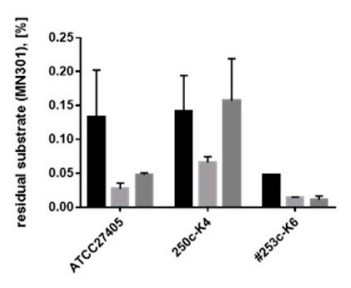

B

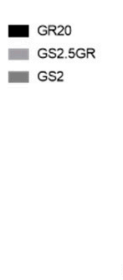

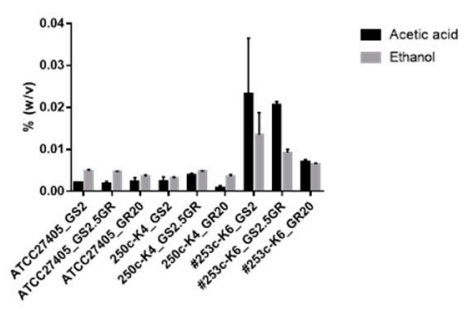

C

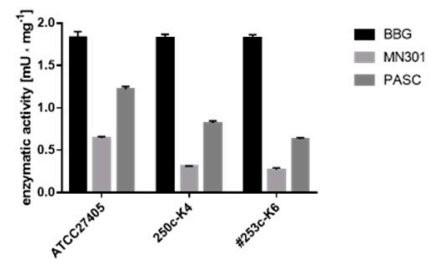

Figure 2. (A) Determination of cellulose hydrolysis for three different cultures with H. thermocellum. (B) Detection of volatile metabolites from three different cultures with $H$. thermocellum via gas chromatography. (C) Specific activity $\left(\mathrm{mU} \cdot \mathrm{mg}^{-1}\right)$ of supernatant proteins on different $\beta$-glucans, soluble (BBG), amorphous (PASC), and crystalline (MN301), from three different cultures with $H$. thermocellum determined by liberation of reducing sugars.

Cultures: ATCC27405 ${ }^{\mathrm{T}}$ and 250c-K4, H. thermocellum alone; 253c-K6, H. thermocellum and D. raffinosedens in co-culture. Co-cultures are highlighted with '\#'.

(A) All cultures were incubated as biological duplicates in three different media (GS2, GS2.5GR and GR20) with a total volume of $50 \mathrm{~mL}$ and $0.2 \%(w / v)$ MN301. Residual MN301 after cultivation was determined by Anthrone assay. Data were normalized to an incubation period of two days. D. raffinosedens alone did not grow on MN301 as sole carbon source and is therefore not included in the figure (residual substrate 100\%).

(B) Metabolite production was compared using three different cultivation media (GS2, GS2.5GR, and GR20) in biological duplicates. Data were normalized to an incubation period of two days at $55^{\circ} \mathrm{C}$. One hundred microliters culture supernatant were measured in a total volume of $500 \mu \mathrm{L}$ with $50 \mu \mathrm{L}$ of $0.5 \%(v / v)$ 1-propanol as internal standard for quantification.

(C) All digests were performed as biological duplicates in a total volume of $200 \mu \mathrm{L}$ with $0.5 \%(w / v)$ substrate in a water bath at $55{ }^{\circ} \mathrm{C}$ for $30 \mathrm{~h}$. Protein concentration within the batch was $0.1 \mu \mathrm{g} \cdot \mu \mathrm{L}^{-1}$. DNSA assay was performed in technical triplicates. Enzymatic activity was calculated with Equation (1):

$$
\text { specific activity }\left(\frac{U}{m l}\right)=\frac{m_{g l u} \times 1000}{M_{g l u} \cdot V_{e} \cdot c_{e} \cdot t}
$$

Equation 1: Calculation of the specific enzymatic activity via DNSA assay. 
$\mathrm{U}$ is units $\left(\mu \mathrm{mol} \times \mathrm{min}^{-1}\right) ; \mathrm{m}_{\mathrm{glu}}$ is the mass of glucose $(\mu \mathrm{g})$ calculated by means of glucose standard curve; $\mathrm{M}_{\mathrm{glu}}$ is the molar mass of glucose $\left(\mu \mathrm{g} \times \mu \mathrm{mol}^{-1}\right)$; $\mathrm{t}$ is the incubation period (min); $\mathrm{V}_{\mathrm{e}}$ is the volume of enzyme within the batch $(\mu \mathrm{L})$; and $c_{e}$ is the concentration of enzyme $\left(\mu g \times \mu \mathrm{L}^{-1}\right)$.

\subsection{Importance of the Genus Defluviitalea in Microbial Communities of Biogas Fermenters}

3.4.1. Comparison of Microbial Community Members' Abundance and Activity as Deduced by $16 \mathrm{~S}$ rDNA and Reverse Transcribed 16S rRNA Amplicon Sequencing

Taxonomic composition and the active part of the microbial biogas communities originating from two thermophilic and mesophilic lab-scale maize-fed biogas reactors operated in parallel (numbered 1 and 2) were examined for the presence of Defluviitalea species in these communities. For this purpose, the hypervariable regions V6-V8 of the bacterial 16S rRNA gene were analyzed in detail by means of $16 \mathrm{~S}$ rRNA (cDNA) and 16S rDNA (DNA) amplicon sequencing (Figure 3). Two mesophilic, stable ( $\left.\mathrm{m} \_s t\right)$ and acidified (m_ac), and two thermophilic, stable ( $\left.\mathrm{t} \_s t\right)$ and highly efficient ( $t \_$ef), process conditions were investigated. The corresponding sequence datasets of the $16 \mathrm{~S}$ rRNA amplicon libraries of this study are available in the ENA repository under the Bioproject ID PRJEB37872.

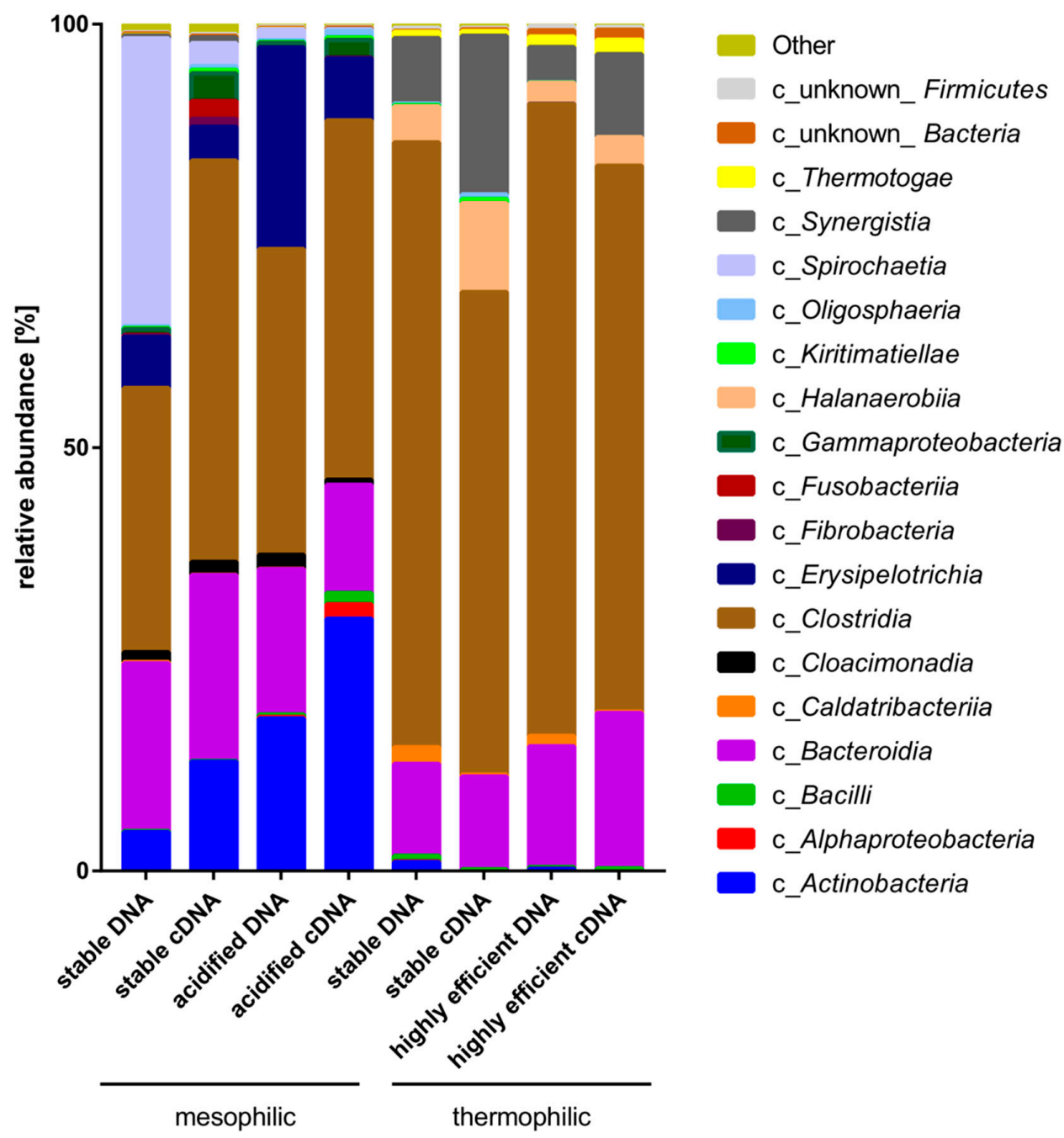

Figure 3. Taxonomic classification at class level of $16 \mathrm{~S}$ rRNA (cDNA) and $16 \mathrm{~S}$ rDNA (DNA) amplicon sequences originating from laboratory-scale of biogas reactors under four different process conditions. Values for the relative abundance at class level were averaged over two biological replicates. 
Looking at DNA, the taxonomic composition at higher taxonomic ranks was highly similar between the fermenter replicates (data not shown). At phylum level, the microbiomes were mainly composed of Firmicutes (on average $74 \%$ in the thermophilic vs. $49 \%$ in the mesophilic processes), followed by Bacteroidetes (on average 13\% in the thermophilic vs. 19\% in the mesophilic processes). In the thermophilic biogas-producing communities, Synergistetes were also significant (on average 6\%), while the mesophilic microbiomes showed high abundance of Actinobateria ( $5 \%$ and $18 \%$ in $\mathrm{m} \_$st and ma_ac, respectively) and, at stable process conditions only, Spirochaetes (on average $34 \%$ in m_st).

Further down in the taxonomic hierarchy, a clear abundance reduction of the class Clostridia was observed under mesophilic conditions ( $31 \%$ and $36 \%$ in m_st and $\mathrm{m} \_$ac, respectively) in comparison to the thermophilic reactors ( $71 \%$ and $75 \%$ in $\mathrm{t} \_s t$ and $\mathrm{t}$ _ef, respectively) (Figure 3). Sequences of the genus Defluviitalea, a member of the class Clostridia, were detected in all thermophilic microbiomes, but less in the DNA samples $t \_s t$ (on average $0.26 \%$ ) than in $t \_e f$ (on average $0.52 \%$ ). Based on DNA analysis, the thermophilic microbiomes were less diverse than the mesophilic microbiomes (e.g., Shannon indices on average 2.84 and 3.4 for thermophilic or mesophilic microbiomes, respectively; for details, see Supplementary Table S6). This is in line with the observed higher species diversity in mesophilic microbial biogas communities, as described previously $[9,63,64]$.

The metabolically active part of the bacterial biogas communities, according to $16 \mathrm{~S}$ rRNA transcript (cDNA) analysis, showed that mainly Firmicutes (58\% in t_st and 65\% in t_ef), Synergistetes (19\% in $\mathrm{t} \_s t$ and $10 \%$ in $\mathrm{t} \_$ef), Bacteroidetes (11\% in $\mathrm{t} \_s t$ and $18 \%$ in $\mathrm{t} \_$ef), and Halanaerobiaeota (11\% in t_st and or $3 \%$ in $\mathrm{t} \_$ef, ) were highly active at thermophilic conditions. At mesophilic conditions, Firmicutes (52\% in $\mathrm{m} \_s t$ and $51 \%$ in $\mathrm{m} \_$ac), Actinobacteria (13\% in $\mathrm{m} \_s t$ and $30 \%$ in $\mathrm{m} \_$ac), and Bacteroides ( $22 \%$ in $\mathrm{m} \_s t$ and $13 \%$ in $\left.\mathrm{m} \_a c\right)$ were transcriptionally most active. Spirochaetes were found to be highly abundant at mesophilic process condition ( $34 \%$ in $\mathrm{m} \_s t \mathrm{DNA}$ ) but showed relatively poor transcription ( $3 \%$ in $\mathrm{m} \_$st cDNA).

Analysis of the $\beta$-diversity (Figure 4 ) revealed significant dissimilarities between the different process conditions in general $(p=0.0001)$. The bacterial composition deduced from DNA and cDNA at the two mesophilic process conditions, m_st and m_ac, differed highly between each other as well as compared to the thermophilic process conditions. In contrast, the two thermophilic process conditions, $t \_s t$ and $t \_$ef, were closely related to each other.

As Defluviitalea spp. were of particular interest, transcriptional activity of this genus in the thermophilic and mesophilic microbiomes was examined in detail. The highest transcriptional activity of the genus Defluviitalea (1.44\%) was observed in the microbiome originating from the thermophilic biogas reactor operating highly efficiently (reactor replicate 2 ). At mesophilic conditions, only a very small portion of cDNA sequences could be classified as belonging to the genus Defluviitalea $(0.07 \%$ at maximum in $\mathrm{m} \_$ac reactor replicate 2; see also Supplementary Table S7). Furthermore, a targeted mapping approach for the organism of interest, namely $D$. raffinosedens was performed to verify the presence of the latter in the community. In total, 468 reads mapped with $>99 \%$ identity to the reference gene [65,66] (full-length 16S rRNA gene; Accession No. MT350287). All hits were observed in DNA or CDNA of the thermophilic reactors only, and not at mesophilic processes (see also Supplementary Table S8). The abundance and transcriptional activity of this species were both higher in $\mathrm{t} \_$ef than in $\mathrm{t} \_s t$, and the relation of the transcripts (cDNA, 321 hits in total) to the genes (DNA, 147 hits in total) is in line with the results on the broader genus level (Supplementary Table S7). 


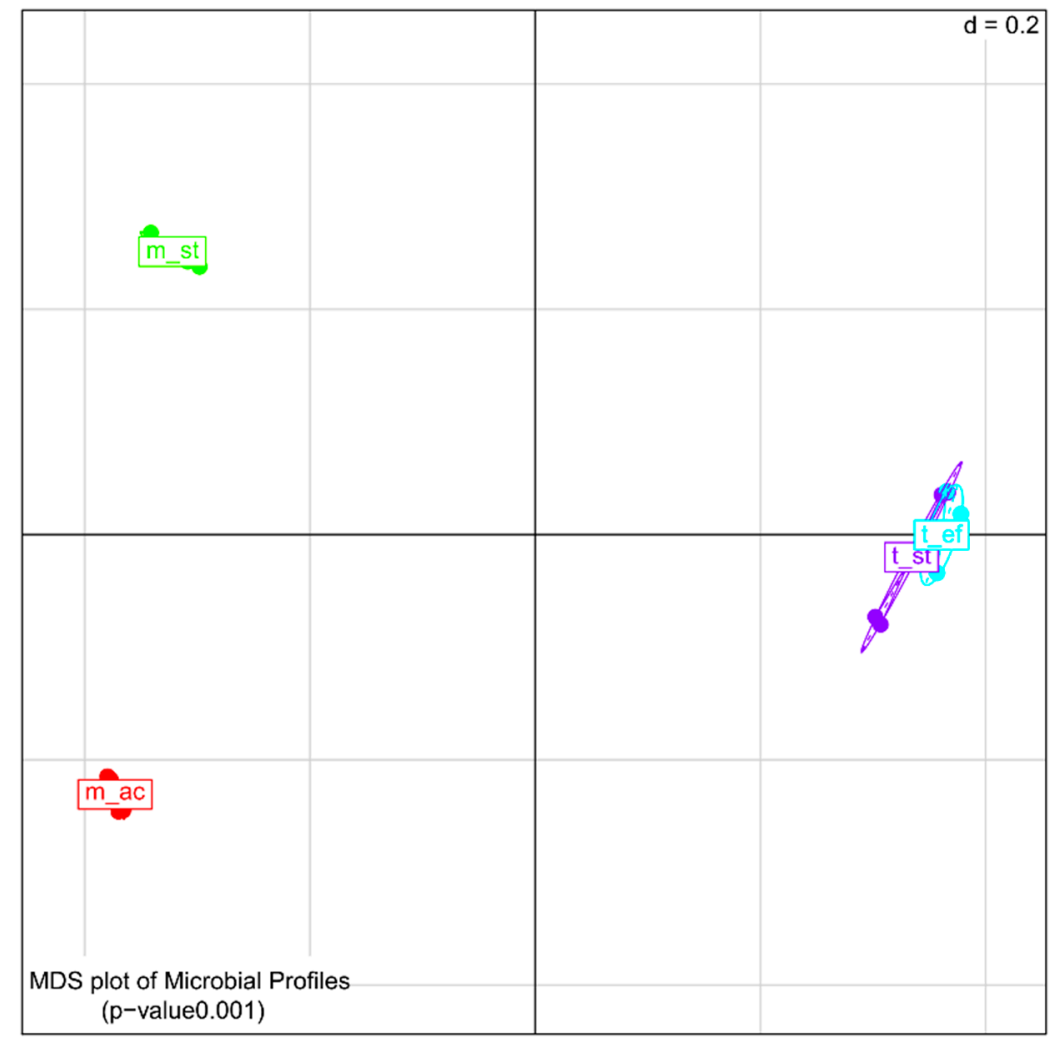

Figure 4. Multi-dimensional scattering (MDS) of the $\beta$-diversity of the microbial profiles derived from 16S rDNA and reverse transcribed 16S rRNA amplicon sequences of biogas reactors under four different process conditions with two biological replicates. Samples were grouped for process condition: m_st, mesophilic $\left(38{ }^{\circ} \mathrm{C}\right)$, stable; m_ac, mesophilic, acidified; t_st, thermophilic $\left(50{ }^{\circ} \mathrm{C}\right)$, stable; t_ef, thermophilic, highly efficient.

We observed a positive correlation between: (i) the abundance of the OTUs representing Defluviitalea spp., the exclusive presence of reads mapped to D. raffinosedens in the thermophilic processes, and a higher number of cDNA than DNA in either case in the sequence libraries; and (ii) the efficiency thermophilic biogas process. This emphasizes the role of this non-cellulolytic organism for thermophilic lignocellulose hydrolysis as already discussed in the previous section. Moreover, the Blastn analysis of the 16S rRNA gene sequence MN744427 against the NCBI nucleotide collection (nr/nt) revealed that of the 21 nucleotide sequences with identities above $98 \%$, eight were related to "biogas" and seven to "cellulose" when analyzing the title of the deposited nucleotide sequence (Supplementary Table S2). This indicates that sequences belonging to the genus Defluviitalea have been detected earlier but could not be assigned to a prokaryote with standing in nomenclature. Thus, identifying $D$. raffinosedens in other publicly available datasets from biogas-related environments (see below) is of great interest.

3.4.2. The Occurrence of Defluviitalea Genus in Biogas-Producing Plants as Deduced from Publicly Available Metagenome Data

A recently published study by Campanaro et al. [53] described the establishment of a repository on microbial genome sequence information, which is of great importance for future studies and broadens our understanding of how the microbes in biogas processes contribute to the anaerobic digestion process. The authors analyzed 134 publicly available metagenome datasets derived from different biogas reactors and recovered 1635 metagenome-assembled genomes (MAGs) representing different bacterial and archaeal species. The genus Defluviitalea was detected in almost all microbiomes analyzed, but differed in abundance values. The highest amount of Defluviitalea was found in the microbiome 
originating from the thermophilic $\left(55^{\circ} \mathrm{C}\right)$ laboratory-scaled packed-bed biogas reactor fed with acetate and yeast extract as the sole sources of carbon and energy [53,67]. In the analysis of the MAGs originating from the corresponding microbiome, the MAG METABAT AS04akNAM 106 was identified as belonging to the genus Defluviitalea (please refer to Additional File 8 of the study by Campanaro et al. [53]). The responsive MAG featured a completeness value of $95.5 \%$ and contamination value being 5.4\%. Hence, an ANI analysis [58], which is suitable for species demarcation [59], was calculated between the MAG METABAT AS04akNAM 106 and D. raffinosedens 249c-K6. The analysis revealed an ANI value of $99.5 \%$ by comparing $96.7 \%$ of the entire MAG sequence, indicating that these two members belong to the same species. Based on the obtained results and the abundance values published previously for Defluviitalea members $[53,68]$, Bacteria highly related to the reference strain D. raffinosedens 249 c-K6 play an important role within the community of the thermophilic biogas plant.

\section{Conclusions}

In this study, metabolic interactions of D. raffinosedens and $H$. thermocellum in respect of microbial conversion of plant-based biomass to biogas were studied. We observed a high frequency of isolating D. raffinosedens in co-culture with $H$. thermocellum and saw higher cellulose hydrolysis as well as a higher production of volatile metabolites for this co-culture in comparison to $H$. thermocellum alone. The results obtained point to a cross-feeding process between $H$. thermocellum and D. raffinosedens. It is proposed that enzymatic activity of $H$. thermocellum liberates soluble mono- and oligosaccharides from cellulose and hemicellulose, thereby promoting growth of the non-cellulolytic, but saccharolytic $D$. raffinosedens. The results obtained clearly indicate that the activity of the latter in turn intensifies cellulose hydrolysis by preventing feedback-inhibition and improving the thermodynamics of the degradation process. Bacterial synergism as observed in this study is supposed to accelerate biomethane production in anaerobic digestion of plant fibers by increasing the overall cellulose hydrolysis rates and increasing the amounts of produced volatile metabolites in a given time. We describe the D. raffinosedens 249c-K6 genome, which is the first of this species and the second within this genus, and therefore provide the first reference sequence of this species. Future studies will certainly benefit from the comprehensive genomic information on D. raffinosedens 249c-K6, e.g. by integrating this knowledge into models describing interactions within corresponding complex communities.

Supplementary Materials: The following are available online at http://www.mdpi.com/2076-2607/8/6/915/s1: Table S1: Chemical analyses of the mesophilic and thermophilic inocula. Table S2: Comparisons of the D. raffinosedens 16S rRNA gene sequence (MN744427) to the sequences of the related organisms deposited in the NCBI nucleotide collection (nr/nt) by means of Blastn and the Megablast algorithm; Table S3: Comparisons of the D. raffinosedens 16S rRNA gene sequence (MN744427) to the sequences of the related organisms deposited in the NCBI reference RNA sequence database (refseq_rna_v5) from type material only by means of Blastn and the Megablast algorithm; Table S4: Summary of 37 complete pathway modules for annotated proteins in the genome of D. raffinosedens 249 c-K6 as identified by means of KEGG (current status January 2020); Table S5: Summary of $D$. raffinosedens carbohydrate active enzymes including signal peptides and their possible activities as listed in the CAZy database (current status August 2019); Table S6: $\alpha$-Diversity of biogas-producing communities originating from two mesophilic and two thermophilic biogas reactors under different process conditions as deduced from 16S rDNA and reverse transcribed 16S rRNA amplicon sequences; Table S7: Relative abundance of the genus Defluviitalea in two mesophilic and two thermophilic biogas reactor sludge at different process conditions; Table S8: Abundance of D. raffinosedens in two mesophilic and two thermophilic biogas reactor sludge at different process conditions.

Author Contributions: Conceptualization, V.Z., M.L., A.S., and W.L.; Methodology, R.R., M.S., B.M., S.J., I.M., and V.Z.; Software, R.R., I.M., and S.J.; Validation, R.R., M.S., B.M., S.J., and I.M.; Formal Analysis, R.R., M.S., B.M., S.J., I.M., and V.Z.; Investigation, R.R., M.S., B.M., S.J., I.M., and V.Z.; Resources, M.L., A.S., and W.L.; Data Curation, R.R., B.M., I.M., and S.J.; Writing-Original Draft Preparation, R.R., M.S., B.M., S.J., I.M., and V.Z.; Writing-Review and Editing, M.L., A.S., and W.L.; Visualization, R.R., M.S., and S.J.; Supervision, M.L., A.S., and W.L.; Project Administration, M.L. and W.L.; and Funding Acquisition, M.L. and W.L. All authors have read and agreed to the published version of the manuscript. 
Funding: Financial support by the German Federal Ministry of Food and Agriculture (grants 22021715 and 22003513) is gratefully acknowledged. The Galaxy server that was used for some calculations is in part funded by Collaborative Research Centre 992 Medical Epigenetics (DFG grant SFB 992/1 2012) and German Federal Ministry of Education and Research (BMBF grants 031 A538A/A538C RBC, 031L0101B/031L0101C de.NBI-epi, $031 \mathrm{~L} 0106$ de.STAIR (de.NBI)). The bioinformatics support of the BMBF-funded project "Bielefeld-Gießen Center for Microbial Bioinformatics-BiGi (Grant No. 031A533)“ within the German Network for Bioinformatics Infrastructure (de.NBI) is also gratefully acknowledged.

Acknowledgments: Next-Generation Sequencing was performed at ZIEL-Core Facility Microbiome, Technical University of Munich, Weihenstephaner Berg 3, 85354 Freising, Germany. The authors thank Klaus Neuhaus for supervision and data analysis and Caroline Ziegler for NGS library preparation.

Conflicts of Interest: The authors declare that there is no conflict of interest.

\section{Abbreviations}

ANI, average nucleotide identity; BBG, barley- $\beta$-glucan; bp, base pairs; CAZymes, Carbohydrate-Active Enzymes; CE, carbohydrate esterase; DNSA, 3,5-dinitrosalicylic acid; FP, filter paper; GC, gas chromatography; GH, glycoside hydrolase; GR, digestate (german: Gärrest); GSI, gene support index; GT, glycosyltransferase; ID, Identification number; KEGG, Kyoto Encyclopedia of Genes and Genomes; MAG, metagenome-assembled genome; MQ, Mili-Q ultrapure water; m_ac, mesophilic_acidified; m_st, mesophilic_stable; OLR, organic loading rate; OTU, operational taxonomic unit; PASC, phosphoric acid swollen cellulose; PE, paired-end; RT, room temperature; SP, signal peptide; taxid, taxonomic identification number; $t \_e f$, thermophilic_highly-efficient; $t \_s t$, thermophilic_stable; UBCG, unique bacterial core genes; VFA, volatile fatty acid.

\section{References}

1. Weiland, P. Biogas production: Current state and perspectives. Appl. Microbiol. Biotechnol. 2010, 85, 849-860. [CrossRef] [PubMed]

2. Lebuhn, M.; Munk, B.; Effenberger, M. Agricultural biogas production in Germany-from practice to microbiology basics. Energy Sustain. Soc. 2014, 4, 10. [CrossRef]

3. Li, K.; Liu, R.; Sun, C. A review of methane production from agricultural residues in China. Renew. Sustain. Energy Rev. 2016, 54, 857-865. [CrossRef]

4. Zverlov, V.V.; Koeck, D.E.; Schwarz, W.H. The role of cellulose-hydrolyzing bacteria in the production of biogas from plant biomass. In Microorganisms in Biorefineries; Springer: Berlin/Heidelberg, Germany, 2015; pp. 335-361.

5. Evans, P.N.; Boyd, J.A.; Leu, A.O.; Woodcroft, B.J.; Parks, D.H.; Hugenholtz, P.; Tyson, G.W. An evolving view of methane metabolism in the Archaea. Nat. Rev. Microbiol. 2019, 17, 219-232. [CrossRef]

6. Bremges, A.; Maus, I.; Belmann, P.; Eikmeyer, F.; Winkler, A.; Albersmeier, A.; Pühler, A.; Schlüter, A.; Sczyrba, A. Deeply sequenced metagenome and metatranscriptome of a biogas-producing microbial community from an agricultural production-scale biogas plant. Gigascience 2015, 4, 33. [CrossRef]

7. Ortseifen, V.; Stolze, Y.; Maus, I.; Sczyrba, A.; Bremges, A.; Albaum, S.P.; Jaenicke, S.; Fracowiak, J.; Pühler, A.; Schlüter, A. An integrated metagenome and-proteome analysis of the microbial community residing in a biogas production plant. J. Biotechnol. 2016, 231, 268-279. [CrossRef]

8. Campanaro, S.; Treu, L.; Kougias, P.G.; De Francisci, D.; Valle, G.; Angelidaki, I. Metagenomic analysis and functional characterization of the biogas microbiome using high throughput shotgun sequencing and a novel binning strategy. Biotechnol. Biofuels 2016, 9, 26. [CrossRef]

9. Maus, I.; Koeck, D.E.; Cibis, K.G.; Hahnke, S.; Kim, Y.S.; Langer, T.; Kreubel, J.; Erhard, M.; Bremges, A.; Off, S.; et al. Unraveling the microbiome of a thermophilic biogas plant by metagenome and metatranscriptome analysis complemented by characterization of bacterial and archaeal isolates. Biotechnol. Biofuels 2016, 9, 171. [CrossRef]

10. Hassa, J.; Maus, I.; Off, S.; Puhler, A.; Scherer, P.; Klocke, M.; Schluter, A. Metagenome, metatranscriptome, and metaproteome approaches unraveled compositions and functional relationships of microbial communities residing in biogas plants. Appl. Microbiol. Biotechnol. 2018, 102, 5045-5063. [CrossRef] [PubMed]

11. Maus, I.; Bremges, A.; Stolze, Y.; Hahnke, S.; Cibis, K.G.; Koeck, D.E.; Kim, Y.S.; Kreubel, J.; Hassa, J.; Wibberg, D.; et al. Genomics and prevalence of bacterial and archaeal isolates from biogas-producing microbiomes. Biotechnol. Biofuels 2017, 10, 264. [CrossRef] [PubMed] 
12. Antoni, D.; Zverlov, V.V.; Schwarz, W.H. Biofuels from microbes. Appl. Microb. Biotechnol. 2007, 77, $23-35$. [CrossRef] [PubMed]

13. Zhu, X.; Campanaro, S.; Treu, L.; Seshadri, R.; Ivanova, N.; Kougias, P.G.; Kyrpides, N.; Angelidaki, I. Metabolic dependencies govern microbial syntrophies during methanogenesis in an anaerobic digestion ecosystem. Microbiome 2020, 8, 1-14. [CrossRef] [PubMed]

14. Fujimoto, M.; Carey, D.E.; Zitomer, D.H.; McNamara, P.J. Syntroph diversity and abundance in anaerobic digestion revealed through a comparative core microbiome approach. Appl. Microbiol. Biotechnol. 2019, 103, 6353-6367. [CrossRef] [PubMed]

15. Levin, D.B.; Verbeke, T.J.; Munir, R.; Islam, R.; Ramachandran, U.; Lal, S.; Schellenberg, J.; Sparling, R. Omics approaches for designing biofuel producing cocultures for enhanced microbial conversion of lignocellulosic substrates. In Direct Microbial Conversion of Biomass to Advanced Biofuels; Elsevier: Amsterdam, The Netherlands, 2015; pp. 335-363.

16. Zhang, X.; Tu, B.; Dai, L.R.; Lawson, P.A.; Zheng, Z.Z.; Liu, L.Y.; Deng, Y.; Zhang, H.; Cheng, L. Petroclostridium xylanilyticum gen. nov., sp. nov., a xylan-degrading bacterium isolated from an oilfield, and reclassification of clostridial cluster III members into four novel genera in a new Hungateiclostridiaceae fam. nov. Int. J. Syst. Evol. Microbiol. 2018, 68, 3197-3211. [CrossRef]

17. Cavedon, K.; Canale-Parola, E. Physiological interactions between a mesophilic cellulolytic Clostridium and a non-cellulolytic bacterium. FEMS Microbiol. Lett. 1992, 86, 237-245. [CrossRef]

18. He, Q.; Hemme, C.L.; Jiang, H.; He, Z.; Zhou, J. Mechanisms of enhanced cellulosic bioethanol fermentation by co-cultivation of Clostridium and Thermoanaerobacter spp. Bioresour. Technol. 2011, 102, 9586-9592. [CrossRef]

19. Kato, S.; Haruta, S.; Cui, Z.J.; Ishii, M.; Igarashi, Y. Stable coexistence of five bacterial strains as a cellulose-degrading community. Appl. Environ. Microbiol. 2005, 71, 7099-7106. [CrossRef]

20. Rettenmaier, R.; Duerr, C.; Neuhaus, K.; Liebl, W.; Zverlov, V.V. Comparison of sampling techniques and different media for the enrichment and isolation of cellulolytic organisms from biogas fermenters. Syst. Appl. Microbiol. 2019, 42, 481-487. [CrossRef]

21. Johnson, E.A.; Madia, A.; Demain, A.L. Chemically defined minimal medium for growth of the anaerobic cellulolytic thermophile Clostridium thermocellum. Appl. Environ. Microbiol. 1981, 41, 1060. [CrossRef]

22. Koeck, D.E.; Ludwig, W.; Wanner, G.; Zverlov, V.V.; Liebl, W.; Schwarz, W.H. Herbinix hemicellulosilytica gen. nov., sp. nov., a thermophilic cellulose-degrading bacterium isolated from a thermophilic biogas reactor. Int. J. Syst. Evol. Microbiol. 2015, 65, 2365-2371. [CrossRef]

23. Miller, G.L. Use of dinitrosalicylic acid reagent for determination of reducing sugar. Anal. Chem. 1959, 31, 426-428. [CrossRef]

24. Koeck, D.E.; Zverlov, V.V.; Liebl, W.; Schwarz, W.H. Comparative genotyping of Clostridium thermocellum strains isolated from biogas plants: Genetic markers and characterization of cellulolytic potential. Syst. Appl. Microbiol. 2014, 37, 311-319. [CrossRef] [PubMed]

25. Huptas, C.; Scherer, S.; Wenning, M. Optimized Illumina PCR-free library preparation for bacterial whole genome sequencing and analysis of factors influencing de novo assembly. BMC Res. Notes 2016, 9, 269. [CrossRef] [PubMed]

26. Nurk, S.; Bankevich, A.; Antipov, D.; Gurevich, A.A.; Korobeynikov, A.; Lapidus, A.; Prjibelski, A.D.; Pyshkin, A.; Sirotkin, A.; Sirotkin, Y.; et al. Assembling single-cell genomes and mini-metagenomes from chimeric MDA products. J. Comput. Biol. 2013, 20, 714-737. [CrossRef]

27. Tatusova, T.; DiCuccio, M.; Badretdin, A.; Chetvernin, V.; Nawrocki, E.P.; Zaslavsky, L.; Lomsadze, A.; Pruitt, K.D.; Borodovsky, M.; Ostell, J. NCBI prokaryotic genome annotation pipeline. Nucleic Acids Res. 2016, 44, 6614-6624. [CrossRef]

28. Kanehisa, M.; Sato, Y.; Morishima, K. BlastKOALA and GhostKOALA: KEGG tools for functional characterization of genome and metagenome sequences. J. Mol. Biol. 2016, 428, 726-731. [CrossRef]

29. Kanehisa, M.; Sato, Y. KEGG Mapper for inferring cellular functions from protein sequences. Protein Sci. 2020, 29, 28-35. [CrossRef]

30. Zhang, H.; Yohe, T.; Huang, L.; Entwistle, S.; Wu, P.; Yang, Z.; Busk, P.K.; Xu, Y.; Yin, Y. dbCAN2: A meta server for automated carbohydrate-active enzyme annotation. Nucleic Acids Res. 2018, 46, W95-W101. [CrossRef]

31. Finn, R.D.; Clements, J.; Eddy, S.R. HMMER web server: Interactive sequence similarity searching. Nucleic Acids Res. 2011, 39, W29-W37. [CrossRef] 
32. Buchfink, B.; Xie, C.; Huson, D.H. Fast and sensitive protein alignment using DIAMOND. Nat. Methods 2015, 12, 59. [CrossRef]

33. Petersen, T.N.; Brunak, S.; Von Heijne, G.; Nielsen, H. SignalP 4.0: Discriminating signal peptides from transmembrane regions. Nat. Methods 2011, 8, 785. [CrossRef] [PubMed]

34. Lombard, V.; Golaconda Ramulu, H.; Drula, E.; Coutinho, P.M.; Henrissat, B. The carbohydrate-active enzymes database (CAZy) in 2013. Nucleic Acids Res. 2013, 42, D490-D495. [CrossRef] [PubMed]

35. Na, S.-I.; Kim, Y.O.; Yoon, S.-H.; Ha, S.-m.; Baek, I.; Chun, J. UBCG: Up-to-date bacterial core gene set and pipeline for phylogenomic tree reconstruction. J. Microbiol. 2018, 56, 280-285. [CrossRef]

36. Richter, M.; Rosselló-Móra, R.; Oliver Glöckner, F.; Peplies, J. JSpeciesWS: A web server for prokaryotic species circumscription based on pairwise genome comparison. Bioinformatics 2016, 32, 929-931. [CrossRef] [PubMed]

37. Lebuhn, M.; Effenberger, M.; Gronauer, A.; Wilderer, P.; Wuertz, S. Using quantitative real-time PCR to determine the hygienic status of cattle manure. Water Sci. Technol. 2003, 48, 97-103. [CrossRef] [PubMed]

38. Lebuhn, M.; Hanreich, A.; Klocke, M.; Schlüter, A.; Bauer, C.; Pérez, C.M. Towards molecular biomarkers for biogas production from lignocellulose-rich substrates. Anaerobe 2014, 29, 10-21. [CrossRef] [PubMed]

39. Engel, K.; Sauer, J.; Jünemann, S.; Winkler, A.; Wibberg, D.; Kalinowski, J.; Tauch, A.; Caspers, B.A. Individual-and species-specific skin microbiomes in three different estrildid finch species revealed by $16 \mathrm{~S}$ amplicon sequencing. Microb. Ecol. 2018, 76, 518-529. [CrossRef] [PubMed]

40. Magoč, T.; Salzberg, S.L. FLASH: Fast length adjustment of short reads to improve genome assemblies. Bioinformatics 2011, 27, 2957-2963. [CrossRef]

41. Joshi, N.; Fass, J. Sickle: A Sliding-Window, Adaptive, Quality-Based Trimming Tool for FastQ Files. 2011. Available online: https://github.com/najoshi/sickle (accessed on 14 December 2019).

42. Martin, M. Cutadapt removes adapter sequences from high-throughput sequencing reads. EMBnet. J. 2011, 17, 10-12. [CrossRef]

43. Schloss, P.D.; Westcott, S.L.; Ryabin, T.; Hall, J.R.; Hartmann, M.; Hollister, E.B.; Lesniewski, R.A.; Oakley, B.B.; Parks, D.H.; Robinson, C.J. Introducing mothur: Open-source, platform-independent, community-supported software for describing and comparing microbial communities. Appl. Environ. Microbiol. 2009, 75, 7537-7541. [CrossRef]

44. Edgar, R.C. Search and clustering orders of magnitude faster than BLAST. Bioinformatics 2010, 26, $2460-2461$. [CrossRef] [PubMed]

45. Quast, C.; Pruesse, E.; Yilmaz, P.; Gerken, J.; Schweer, T.; Yarza, P.; Peplies, J.; Glöckner, F.O. The SILVA ribosomal RNA gene database project: Improved data processing and web-based tools. Nucleic Acids Res. 2013, 41, D590-D596. [CrossRef] [PubMed]

46. Nawrocki, E.P.; Kolbe, D.L.; Eddy, S.R. Infernal 1.0: Inference of RNA alignments. Bioinformatics 2009, 25, 1335-1337. [CrossRef] [PubMed]

47. Stamatakis, A. RAxML version 8: A tool for phylogenetic analysis and post-analysis of large phylogenies. Bioinformatics 2014, 30, 1312-1313. [CrossRef]

48. Lagkouvardos, I.; Fischer, S.; Kumar, N.; Clavel, T. Rhea: A transparent and modular R pipeline for microbial profiling based on 16S rRNA gene amplicons. PeerJ 2017, 5, e2836. [CrossRef]

49. Li, H.; Durbin, R. Fast and accurate short read alignment with Burrows-Wheeler transform. Bioinformatics 2009, 25, 1754-1760. [CrossRef]

50. Yoon, S.-H.; Ha, S.-M.; Kwon, S.; Lim, J.; Kim, Y.; Seo, H.; Chun, J. Introducing EzBioCloud: A taxonomically united database of $16 \mathrm{~S}$ rRNA gene sequences and whole-genome assemblies. Int. J. Syst. Evol. Microbiol. 2017, 67, 1613. [CrossRef]

51. Li, H.; Handsaker, B.; Wysoker, A.; Fennell, T.; Ruan, J.; Homer, N.; Marth, G.; Abecasis, G.; Durbin, R. 1000 Genome Project Data Processing Subgroup. 2009. The sequence alignment/map format and samtools. Bioinformatics 2009, 25, 2078-2079. [CrossRef]

52. Rodriguez-R, L.M.; Gunturu, S.; Harvey, W.T.; Rosselló-Mora, R.; Tiedje, J.M.; Cole, J.R.; Konstantinidis, K.T. The Microbial Genomes Atlas (MiGA) webserver: Taxonomic and gene diversity analysis of Archaea and Bacteria at the whole genome level. Nucleic Acids Res. 2018, 46, W282-W288. [CrossRef] 
53. Campanaro, S.; Treu, L.; Rodriguez-R, L.M.; Kovalovszki, A.; Ziels, R.M.; Maus, I.; Zhu, X.; Kougias, P.G.; Basile, A.; Luo, G. New insights from the biogas microbiome by comprehensive genome-resolved metagenomics of nearly 1600 species originating from multiple anaerobic digesters. Biotechnol. Biofuels 2020, 13, 1-18. [CrossRef]

54. Ma, S.; Huang, Y.; Wang, C.; Fan, H.; Dai, L.; Zhou, Z.; Liu, X.; Deng, Y. Defluviitalea raffinosedens sp. nov., a thermophilic, anaerobic, saccharolytic bacterium isolated from an anaerobic batch digester treating animal manure and rice straw. Int. J. Syst. Evol. Microbiol. 2017, 67, 1607. [CrossRef] [PubMed]

55. Koeck, D.; Koellmeier, T.; Zverlov, V.; Liebl, W.; Schwarz, W. Differences in biomass degradation between newly isolated environmental strains of Clostridium thermocellum and heterogeneity in the size of the cellulosomal scaffoldin. Syst. Appl. Microbiol. 2015, 38, 424-432. [CrossRef] [PubMed]

56. Jabari, L.; Gannoun, H.; Cayol, J.L.; Hamdi, M.; Fauque, G.; Ollivier, B.; Fardeau, M.L. Characterization of Defluviitalea saccharophila gen. nov., sp. nov., a thermophilic bacterium isolated from an upflow anaerobic filter treating abattoir wastewaters, and proposal of Defluviitaleaceae fam. nov. Int. J. Syst. Evol. Microbiol. 2012, 62, 550-555. [CrossRef] [PubMed]

57. Ji, S.Q.; Wang, B.; Lu, M.; Li, F.L. Defluviitalea phaphyphila sp. nov., a Novel Thermophilic Bacterium That Degrades Brown Algae. Appl. Environ. Microbiol. 2016, 82, 868-877. [CrossRef]

58. Konstantinidis, K.T.; Tiedje, J.M. Genomic insights that advance the species definition for prokaryotes. Proc. Natl. Acad. Sci. USA 2005, 102, 2567-2572. [CrossRef]

59. Tindall, B.J.; Rosselló-Móra, R.; Busse, H.-J.; Ludwig, W.; Kämpfer, P. Notes on the characterization of prokaryote strains for taxonomic purposes. Int. J. Syst. Evol. Microbiol. 2010, 60, 249-266. [CrossRef]

60. Koendjbiharie, J.G.; Wiersma, K.; van Kranenburg, R. Investigating the central metabolism of Clostridium thermosuccinogenes. Appl. Environ. Microbiol. 2018, 84, e00363-18. [CrossRef]

61. Ng, T.K.; Ben-Bassat, A.; Zeikus, J. Ethanol production by thermophilic bacteria: Fermentation of cellulosic substrates by cocultures of Clostridium thermocellum and Clostridium thermohydrosulfuricum. Appl. Environ. Microbiol. 1981, 41, 1337-1343. [CrossRef]

62. Zhang, Y.H.; Lynd, L.R. Cellulose utilization by Clostridium thermocellum: Bioenergetics and hydrolysis product assimilation. Proc. Natl. Acad. Sci. USA 2005, 102, 7321-7325. [CrossRef]

63. Lee, S.-H.; Kang, H.-J.; Lee, Y.H.; Lee, T.J.; Han, K.; Choi, Y.; Park, H.-D. Monitoring bacterial community structure and variability in time scale in full-scale anaerobic digesters. J. Environ. Monit. 2012, 14, 1893-1905. [CrossRef]

64. Sundberg, C.; Al-Soud, W.A.; Larsson, M.; Alm, E.; Yekta, S.S.; Svensson, B.H.; Sørensen, S.J.; Karlsson, A. 454 pyrosequencing analyses of bacterial and archaeal richness in 21 full-scale biogas digesters. FEMS Microbiol. Ecol. 2013, 85, 612-626. [CrossRef] [PubMed]

65. Edgar, R.C. Updating the $97 \%$ identity threshold for $16 S$ ribosomal RNA OTUs. Bioinformatics 2018, 34, 2371-2375. [CrossRef] [PubMed]

66. Johnson, J.S.; Spakowicz, D.J.; Hong, B.Y.; Petersen, L.M.; Demkowicz, P.; Chen, L.; Leopold, S.R.; Hanson, B.M.; Agresta, H.O.; Gerstein, M.; et al. Evaluation of 16S rRNA gene sequencing for species and strain-level microbiome analysis. Nat. Commun. 2019, 10, 5029. [CrossRef] [PubMed]

67. Kouzuma, A.; Tsutsumi, M.; Ishii, S.I.; Ueno, Y.; Abe, T.; Watanabe, K. Non-autotrophic methanogens dominate in anaerobic digesters. Sci. Rep. 2017, 7, 1-13. [CrossRef]

68. Maus, I.; Klocke, M.; Derenkó, J.; Stolze, Y.; Beckstette, M.; Jost, C.; Wibberg, D.; Blom, J.; Henke, C.; Willenbücher, K. Impact of process temperature and organic loading rate on cellulolytic/hydrolytic biofilm microbiomes during biomethanation of ryegrass silage revealed by genome-centered metagenomics and metatranscriptomics. Environ. Microbiome 2020, 15, 1-21. [CrossRef]

(C) 2020 by the authors. Licensee MDPI, Basel, Switzerland. This article is an open access article distributed under the terms and conditions of the Creative Commons Attribution (CC BY) license (http://creativecommons.org/licenses/by/4.0/). 